\title{
Ideological versus Downsian political competition
}

\section{Ignacio Ortuño Ortín}

Department of Economics and I.V.I.E., University of Alicante, Ap. Correos 99, 03080 Alicante, Spain (e mail: ortin@merlin.fae.ua.es)

\begin{abstract}
We analyze a one-dimensional model of spatial political competition with two parties and uncertainty on the distribution of voters' types. We assume that parties are formed by regular members and professional politicians; members care about the policy enacted, while professional politicians, on the contrary, care only about winning the election. We consider two possibilities: that members choose the political platforms and that professional politicians are the ones who choose such platforms. The expected utility for party members is analyzed under these two cases. We find that when professional politicians have no informational advantage, it is on the interest of both parties to let professional politicians choose the platforms. Only in the case in which professional politicians have much better information than the members of the party about voters is it possible that party members obtain a greater expected utility choosing the platforms themselves rather than letting the professional politicians choose.
\end{abstract}

\section{Introduction}

The traditional view in models of spatial political competition assumes that parties are non-ideological organizations run by professional politicians who try to maximize the probability of winning the election (see Downs [9]). Many

\footnotetext{
A first version of this paper was written while visiting the Economics Department at Stanford University. I am grateful to Klaus Desmet, Anke Gerber, Peter Hammond, Martin Peitz, Socorro Puy and Fernando Vega for helpful comments. I am particularly indebted to Subir Chattopadhyay and Ramon Fauli for suggesting how to shorten the proof of the main theorem. I wish to acknowledge financial support from the Spanish Ministry of Educacion, projects no. PB94 1504, PB93 0342, PB97 0131 and D.G.I.C.T. mobility grant.
} 
recent papers, however, take a different position which supposes that political competition is best described by ideological party competition (see for example Wittman [17]; Calvert [5]; Alesina [1]; Roemer [15, 16]; Alesina and Rosenthal $[3,2]$; Ortuno-Ortín [12] and the survey in Osborne [13]). In this case parties have preferences on the policy space and their goal is not to win the election per se but to implement the best possible policy according to those preferences.

In models with non-ideological parties (Downsian models) the general result, for the two party case, is that at equilibrium the policy proposals converge. Under the alternative approach of ideological parties, and assuming uncertainty, the typical result is that the policy proposals do not converge (see Roemer $[15,16]$.)

This problem of the objective function of parties is, in certain aspects, parallel to the much studied question in economics of the objective of the firm. We can see members and supporters of a party as the shareholders of a firm and professional candidates or politicians as the managers. Managers' goals differ from those of the shareholders. One of the problems faced by shareholders is how to control the actions taken by managers. It is common to assume that managers have better information about some relevant economic variables than shareholders. In our problem it will be assumed that professional politicians have better information about the electorate than the general members of the party.

In this paper we consider a very simple model of political competition with two parties which can be run either by the members or by professional politicians. We will identify the members with the party and the professional politicians with the candidate. Parties will have well defined preferences on the set of feasible policies. Candidates, on the contrary, only care about winning the election and they may have better information than the parties about the (uncertain) distribution of voters.

Two main scenarios will be analyzed: i) The two parties directly compete between themselves and candidates don't play any role. This would be the case in which, for example, the party general assembly determines the political platform and the candidate is a perfect agent of the party ${ }^{1}$. ii) In the second scenario we consider the "Downsian" case in which competition takes place between the candidates. Candidates don't care about the policy that is enacted, they just want to win the election. Here party members will have no influence on the political proposals ${ }^{2}$.

Our objective is to compare the ex-ante expected utility (i.e., the expected utility before the outcome of the election is known) that parties will get under these two cases. The results will depend on the extent of asymmetry of infor-

\footnotetext{
1 A party is formed by many members with possibly different preferences. We assume, however, that they have already solved their preference aggregation problem so that the party has well defined preferences. These preferences could coincide, for example, with the preferences of the median member of the party or the median constituency.

2 Section 4 provides some comments on a more realistic scenario in which candidates can still choose the political platforms, as in ii). Parties, however, are able to impose some bounds on the set of policy platforms that candidates can adopt.
} 
mation between candidates and parties. In the case in which such an asymmetry does not exist, so that candidates and parties have the same information about the (uncertain) distribution of voters' preferences, we show that both parties are, ex-ante, better off if candidates run the party, i.e., they are better off in case ii) than in cases i). The intuition is simple. If parties compete among themselves and there is uncertainty about the distribution of voters, at equilibrium there is no convergence and, therefore, the outcome is a lottery. Sometimes the outcome is a "leftist" policy and sometimes is a "conservative" policy. In the case of candidate competition, on the contrary, there is always convergence to the expected median voter ideal policy which is a "moderate" policy. If parties are risk-averse this moderate policy is preferred by both parties to the expected outcome under direct party competition. Thus, our result will show not only that the outcome in case ii) is less extreme than the outcome in i), but also that both ideological parties are better off under ii) than under i). However, when candidates have much better information than parties about the distribution of voters' preferences this insurance effect may disappear. Namely, we will show that in case ii) the expected utility for parties is decreasing in the "amount" of information candidates have about the distribution of voters.

If we accept the view that in most advanced democracies professional politicians and the general population share the same basic information about the distribution of voters, ${ }^{3}$ then our results suggest that competition between professional politicians, who only want to be in office, may be an efficient way to insure parties (and voters) against "radical" changes in policy.

\section{The basic model}

In this and the next sections we model political competition as a game played by the two parties. Candidates play no role here and will only be introduced in Sect. 4. Parties have well defined utility functions on the space of feasible policies.

Let $\mathfrak{R}$ be the set of agents' types. Agent of type $\omega \in \mathfrak{R}$ has von NeumannMorgenstern utility function $u(. ; \omega): \mathfrak{R} \rightarrow \mathfrak{R}$, where $u$ is a concave function and $\omega$ is the ideal point of type $\omega$, i.e., $\omega$ is such that $u(\omega ; \omega)>u(x ; \omega)$ for all $x \neq \omega$. We assume that $u(x ; \omega)$ is symmetric about $\omega$, that is, for all $x \in \mathfrak{R}$ we have $u(\omega-x ; \omega)=u(\omega+x ; \omega)^{4}$. To make some proofs simpler we also assume that $u($.$) is twice continuously differentiable.$

${ }^{3}$ For example, the most prestigious opinion polls are always published in the general press. This, however, doesn't always imply that the general population and professional politicians share the same relevant information about the distribution of voters. The opinions that matter are opinions on policy and most of the polls published in the gen eral press don't provide information on this.

4 Under symmetric preferences, an agent always votes for the proposal closest to her ideal policy. This assumption makes the whole analysis simpler and more transparent. Eaton and Lipsey [10], Denzau et al. [8], Cox [7], for example, also consider these pref erences. 
The two parties are denoted by $L$ and $R$. Party $L(R)$ has utility function $u_{L}: \mathfrak{R} \rightarrow \mathfrak{R}\left(u_{R}: \mathfrak{R} \rightarrow \mathfrak{R}\right)$ that coincides with the utility function of agent of type $\omega=0(\omega=1)$, i.e., $u_{L}(x)=u(x ; 0)$ for all $x \in \mathfrak{R}\left(u_{R}(x)=u(x ; 1)\right.$ for all $x \in \mathfrak{R})$. The political game is such that each party announces (simultaneously) a policy which must be an element of $\mathfrak{R}$. Each agent votes for the party that announced the policy which gives a higher utility according to her utility function. Let $l \in \mathfrak{R}(r \in \mathfrak{R})$ be the policy announced by party $L(R)$. Given the pair of proposals $(l, r)$, the agent of type $\omega$ votes for $L(R)$ if $u(l ; \omega)>u(r ; \omega)$ (if $u(l ; \omega)<u(r ; \omega))$. If $u(l ; \omega)=u(r ; \omega)$ she votes for party $L$ with probability $1 / 2 .{ }^{5}$ It is not difficult to show that, given $(l, r)$, the agent of type $\omega_{d}(l, r):=\frac{l+r}{2}$ is indifferent between $l$ and $r$ and all agents with type $\omega<\omega_{d}(l, r)\left(\omega>\omega_{d}(l, r)\right)$ will vote for party $L(R)$. The existence of this "dividing" type $\omega_{d}(l, r)$ will simplify some of the proofs. If $l \neq r$ party $L(R)$ will win the election whenever $\omega_{d}(l, r)$ is greater (less) than the median type. In the case both parties announce the same policy, i.e. $l=r=\omega_{d}(l, r)$, party $L$ wins with probability 0.5 . Note that parties need information only about the location of the type containing the median voter.

We suppose that parties are uncertain about the distribution of voters' types (see Roemer [15, 16]; Wittman [17, 18]; Hansson and Stuart [11]). Namely, parties are uncertain about the median type. They believe that the median type is a random variable distributed according to the density function $f: \mathfrak{R} \rightarrow \mathfrak{R}_{+}$. This function is unimodal and symmetric with respect to the point $1 / 2$ and therefore $f$ attains its maximum at $1 / 2$.

Assumption 1. The density function $f$ is unimodal and satisfies

1. $f(x)=0$ if $x \notin[0,1]$

2. $f\left(\frac{1}{2}-k\right)=f\left(\frac{1}{2}+k\right)$ for all $k \in\left[0, \frac{1}{2}\right]$

The expected median type is 0.5 . Parties are "located" around such an expected value. The actual median type, however, can deviate in one direction or the other from 0.5 . We assume that such deviations are equally probable in either direction. Moreover, the ideal policy of the median voter is always in between the ideal policy of party $R$ and the ideal policy of party $L$. Hence the ideal policy of the median voter will be an element in the set $\Omega=[0,1] .{ }^{6} \mathrm{We}$ will denote by $F$ the cumulative density function of $f$.

Let $\pi(l, r)$ be the probability that party $L$ wins. If $l=r$ we have $\pi(l, r)=\frac{1}{2}$. If $l<r$ we have that $\pi(l, r)$ is the probability that the dividing type $\omega_{d}(l, r)$ is greater than the median type, i.e. $\pi(l, r)=F\left(\omega_{d}(l, r)\right)$.

\section{The party equilibrium}

In this section we analyze the equilibrium of the game played by the two parties when candidates have no influence on the choice of strategies. If party $L$ and

5 The set of agents that are indifferent between two policies has measure zero.

6 This restriction is imposed for simplicity and our results also hold for the more gen eral case in which the support of $f$ is the interval $[0.5 \quad M, 0.5+M], M>0$. 
party $R$ propose $l$ and $r, l \leq r$, respectively, the expected utilities of parties are given by

$$
\begin{aligned}
& v_{L}(l, r) \equiv \pi(l, r) u_{L}(l)+(1-\pi(l, r)) u_{L}(r) \\
& v_{R}(l, r) \equiv \pi(l, r) u_{R}(l)+(1-\pi(l, r)) u_{R}(r)
\end{aligned}
$$

A Party Equilibrium (PE) is a pair of proposals $\left(l^{p}, r^{p}\right)$ which is a Nash equilibrium of the game played by the parties with the payoff functions given by (1) and (2).

The next assumption will be used to establish the existence of a PE.

Assumption 2. (Log-concavity of $F$ and $1-F$ ). The ratio $\frac{f(x)}{F(x)}$ is non increasing and the ratio $\frac{f(x)}{1 F(x)}$ is non decreasing in $x .^{7}$

We say that a Party Equilibrium $\left(l^{p}, r^{p}\right)$ is symmetric if $l^{p}=1-r^{p}$. The following lemma shows the existence of a unique symmetric Party Equilibrium. ${ }^{8}$

Lemma 1. Let Assumptions 1 and 2 hold. Then there exists a unique symmetric Party Equilibrium, $\left(l^{p}, r^{p}\right)$. Furthermore, $l^{p}<r^{p}$.

All the proofs are provided in the Appendix.

We will denote by $\bar{v}_{L}\left(\bar{v}_{R}\right)$ the expected utility for party $L(R)$ in the Party Equilibrium, i.e., $\bar{v}_{L}=v_{L}\left(l^{p}, r^{p}\right)$ and $\bar{v}_{R}=v_{R}\left(l^{p}, r^{p}\right)$.

\section{Competition with Downsian candidates}

We now assume that both parties are run by professional politicians which we call candidates. To make things simpler it is assumed that each party is run by a unique candidate. Candidates are not ideological, that is, they do not have preferences on the political issues. Their objective is to maximize the probability of winning the election.

In this section we analyze the special case in which parties have no means to control the policy adopted by the candidates. Our task will be to compare

\footnotetext{
7 This assumption is weaker than concavity of $\log f$ (see Barlow and Proschan [4]), i.e., if $f$ is $\log$ concave then $F$ is $\log$ concave, but $\log$ concavity of $F$ does not imply $\log$ concavity of $f$. Most commonly used density functions are log concave and therefore satisfy our assumption. Concavity of $\log f$ implies unimodality but concavity of $\log F$ does not. Thus the unimodality condition stated in Assumption 1 is not superfluous. See Caplin and Nalebuff [6] for a list of the class of log concave densities and Pratt [14] for examples of distribution functions for which $\log F$ and $\log \left(\begin{array}{ll}1 & F\end{array}\right)$ are concave but $\log f$ is not.

8 Unless we impose further restrictions we cannot rule out the existence of additional non symmetric equilibria. In Roemer [16], existence of equilibrium is proven under a joint condition on the utility function of the voters and on $F$ which is stronger than our Assumption 2. The reason why we need a weaker condition is that here the "dividing" type is given by $\frac{l+r}{2}$ and there the dividing type is given by a different function which depends on the curvature of the agents' utility functions.
} 
the expected utility of parties under the equilibrium obtained in the previous section with their expected utility if candidates run the parties.

We want to model the idea that candidates may have better information than parties about the distribution of the median voter. This better information will be described by a (measurable) partition $P$ of $\Omega=[0,1]$. Thus, if candidates have the "information partition" $P$ and $\bar{x}$ is the true median type, candidates know that the true median type is in the event in $P$ which contains $\bar{x}$. We will denote by $P_{p}$ the information partition of the parties. This partition is such that $P_{p}=\{\Omega\}$. Thus parties only know that the median type is in the set $\Omega$ and that it is distributed according to the cumulative distribution function $F$. Candidates may have an information partition $P$ different from $P_{p}$. We will also be interested in what happens when the information held by candidates "improves". Improvements in information will be represented by finer partitions of $\Omega$.

More formally, let $P_{n}, n=1,2,3 \ldots$, be the partition of $\Omega$ formed by $2^{n} 1$ intervals such that

$$
P_{n}=\left\{\left[a_{0}^{n}, a_{1}^{n}\right),\left[a_{1}^{n}, a_{2}^{n}\right), \ldots,\left[a_{2^{n}}^{n} 1_{1}, a_{2^{n}} 1\right]\right\}
$$

where $a_{0}^{n}=0, a_{2^{n} 1}^{n}=1$, and $a_{i}^{n}<a_{i+1}^{n}$.

We also write the ith element of $P_{n}$ as $I_{i}^{n}$ so that $P_{n}=\left\{I_{1}^{n}, I_{2}^{n}, \ldots, I_{2^{n}}^{n}\right\}$. Given the partition $P_{n}$, and in order to simplify the notation, we write $o(i) \equiv$ $2^{n} 1-i+1$.

Assumption 3. i) For all $n$ and for all $i$ we have

$$
I_{i}^{n}=I_{2 i}^{n+1} \cup I_{2 i}^{n+1}
$$

ii) For all $n>1$ and for all $i \leq \frac{1}{2} 2^{n} 1^{1}$ we have that the interval $I_{i}^{n}=\left[a_{i 1}^{n}, a_{i}^{n}\right)$ and the interval $I_{o(i)}^{n}=\left[a_{o(i)}^{n}, a_{o(i)}^{n}\right)$ are symmetric around point $\frac{1}{2}$, i.e.

$$
a_{o(i)}^{n}=1-a_{i 1}^{n} \quad \text { and } a_{o(i)}^{n} 1=1-a_{i}^{n}
$$

The first part of the assumption says that if $n^{\prime}>n$ then $P_{n^{\prime}}$ is a finer partition than $P_{n}$. Thus, partition $P_{n^{\prime}}$ provides a better information about the distribution of the median voter than the information provided by partition $P_{n}$. The second part of the assumption states that an information partition can have no bias towards one of the "sides", i.e. each element of the partition lying on the left hand side of $\frac{1}{2}$ has its identical counterpart on the right hand side of $\frac{1}{2}$.

If candidates have information partition $P_{1}$, they have the same information as parties (that is, $P_{p}=P_{1}=\{\Omega\}$ ). We will denote by $P_{\infty}$ the full information case, that is, the case in which candidates know the true value of the median type.

Candidates, as well as parties, know that the prior distribution for the median type is given by the cumulative density function $F$ with density function $f$. However, they also have the information partition $P_{n}$. Before announcing their policies both candidates observe a signal $s_{j}^{n}, j \in\left\{1,2, \ldots 2^{n}{ }^{1}\right\}$ the same signal for both candidates that indicates the element $I_{j}^{n}$ of the partition $P_{n}$ in 
which the true median type lies. After observing this signal candidates update the prior to obtain the posterior density function $f\left(x \mid I_{j}^{n}\right)$. This posterior is calculated by Bayes' rule, that is

$$
f\left(x \mid I_{j}^{n}\right)= \begin{cases}\frac{f(x)}{\int_{I_{j}^{n}} f(y) d y} & \text { for all } x \in I_{j}^{n} \\ 0 & \text { otherwise }\end{cases}
$$

We write the cumulative density function associated with $f\left(. \mid I_{j}^{n}\right)$ as $F\left(. \mid I_{j}^{n}\right)$. We also write the median of $F\left(. \mid I_{j}^{n}\right)$ as $m_{j}^{n}$, i.e., $F\left(m_{j}^{n} \mid I_{j}^{n}\right)=\frac{1}{2}$.

The idea is that before receiving the signal candidates have the same information as parties (this information is captured by the prior $f$.) Candidates, however, have the advantage of being able to observe a signal which provides more information about the value of the median type.

The political game is such that, after observing signal $s_{j}^{n}$, each candidate announces (simultaneously) a policy. Each agent in a similar way to our assumption in the previous section votes for the candidate that announced the policy that gives higher utility according to her utility function. Let $a \in \mathfrak{R}$ $(b \in \mathfrak{R})$ be the policy announced by candidate of party $L(R)$. As in the previous sections we write the "dividing" type as $\omega_{d}(a, b)=\frac{a+b}{2}$. Let $\Pi_{L}\left(a, b ; I_{j}^{n}\right)$ $\left(\Pi_{R}\left(a, b ; I_{j}^{n}\right)\right)$ be the probability of winning for the candidate of party $L(R)$ when the information partition is $P_{n}$, the signal is $s_{j}^{n}$ and the announced policies are $(a, b)$, that is, for $a \neq b$

$$
\begin{aligned}
& \Pi_{L}\left(a, b ; I_{j}^{n}\right)=F\left(\frac{a+b}{2} \mid I_{j}^{n}\right) \\
& \Pi_{R}\left(a, b ; I_{j}^{n}\right)=1-F\left(\frac{a+b}{2} \mid I_{j}^{n}\right)
\end{aligned}
$$

and for $a=b, \Pi_{L}\left(a, b ; I_{j}^{n}\right)=\Pi_{R}\left(a, b ; I_{j}^{n}\right)=\frac{1}{2}$.

A Candidate Equilibrium for the information partition $P_{n}$ and the signal $s_{j}^{n}$ is a pair of proposals $\left(a^{*}, b^{*}\right)$ which is a Nash equilibrium of the game played by the candidates with the payoff functions given by (3) and (4).

The following lemma states that the proposals of the candidates converge to the median value of the ideal policy of the median voter. Thus, this is a kind of "median voter theorem" with uncertainty.

Lemma 2. Let candidates have information partition $P_{n}$ and observe signal $s_{j}^{n}$. Then, the unique Candidate Equilibrium is given by $a^{*}=b^{*}=m_{j}^{n}$.

Notice that for partition $P_{1}$ the candidate equilibrium is such that $a^{*}=$ $b^{*}=\frac{1}{2}$. Our goal now is to analyze the expected utility of parties when candidates play the political game. It is assumed that parties know $P_{n}$, the information partition of the candidates. What parties don't know is the signal observed by candidates. They, however, associate probabilities to each possible signal. Given information partition $P_{n}$ parties believe that candidates will observe signal $s_{j}^{n}$ with probability $e_{j}^{n} \equiv \int_{I_{j}^{n}} f(x) d x$. Thus, the probability $e_{j}^{n}$ is consistent with the prior $f$. Parties also know that if candidates observe signal 
$s_{j}^{n}$ they will adopt the policy given by the corresponding candidate equilibrium. Thus, the expected utility for parties when candidates play the political game and have information partition $P_{n}$ is given by

$$
\begin{aligned}
& v_{L}^{c}(n) \equiv \sum_{j=1}^{2^{n} 1} e_{j}^{n} u_{L}\left(m_{j}^{n}\right) \\
& v_{R}^{c}(n) \equiv \sum_{j=1}^{2^{n} 1} e_{j}^{n} u_{R}\left(m_{j}^{n}\right)
\end{aligned}
$$

Next we compare the expected utility of parties under the Candidate Equilibrium with the utility they get under the Party Equilibrium obtained in Sect. 3. This comparison clearly depends on the specific information partition that candidates have, that is, it depends on how much information advantage candidates have. Recall that $\bar{v}_{i}$ is the utility party $i$ gets under the Party Equilibrium.

Theorem 3. Let Assumptions 1 and 2 hold. We have: i) If parties and candidates share the same information the expected utility, for both parties, under the Candidate Equilibrium is greater than the expected utility under the Party Equilibrium. ii) In the case candidates have better information than the parties, the expected utility parties get from the Candidate Equilibrium decreases as the informational advantage of candidates over parties increases. Formally for $i=L, R$

i) $\bar{v}_{i}<v_{i}^{c}(1)$;

ii) $v_{i}^{c}(n)$, is decreasing in $n$;

The theorem shows that if candidates have no information advantage then both parties are better off under the Candidate Equilibrium than under the Party Equilibrium. In the Party Equilibrium, proposals don't converge and, as a consequence, both parties get involved in a lottery. Under the Candidate Equilibrium, on the contrary, proposals converge. Thus, candidates can be seen as a device to lower the risk that would be created by direct competition of the two ideological parties. However, it is important to emphasize that this result does not imply that the members of a party prefer that it be run by a candidate who is interested only in winning. In fact, a party is not better off if unilaterally lets its candidate choose the policy proposal ${ }^{9}$. Thus, the theorem only analyzes the welfare implications of two exogenously given political competition settings. One might consider a richer model than the one developed

\footnotetext{
9 Say that only party $L$ delegates policy selection to its candidate. In this case, party $R$ would propose policy $r=1$ (its ideal policy) and the candidate of $L$ wants to propose a policy $l<1$ "as close as possible" to 1 . Thus, candidate $L$ 's best reply is not well defined. However, this is just a technical problem and we can assume that the candidate of party $L$ would propose a policy that only slightly differs from $r$. It is clear that here the ex pected utility for party $L$ is less than under the Party Competition equilibrium.
} 
in this paper in which these settings are endogenous. For instance, consider a two-period game in which in the first period parties choose (simultaneously) whether or not to "delegate" on candidates. In the second period either the party or the candidate, depending on the first period choice, chooses policy and the election takes places. It is not difficult to see that under some technical additional assumptions ${ }^{10}$ the unique (subgame perfect) Nash equilibrium of this game implies no delegation and, as a consequence, the payoff outcome coincides with the utilities obtained under the Party Equilibrium of our model. Thus, the parties' decisions to delegate policy selection to the candidates is a sort of prisoners' dilemma.

The second part of the theorem states that the expected utility parties get from the Candidate Equilibrium decreases as the informational advantage of candidates over parties increases. To get an intuition for this result think, for example, of the full information case $P_{\infty}$ : parties know that the Candidate Equilibrium coincides with the true median type which can be any policy in $[0,1]$ (with density function $f$ ). Thus, letting candidates play the political game might not be an "insurance device" any more. Even tough in general we cannot compare $v_{i}^{c}(\infty)$ and $\bar{v}_{i}$ one can easily provide examples in which $v_{i}^{c}(\infty) \leq \bar{v}_{i}$ for $i=L, R$. In this case, both parties are better off if they, instead of the candidates, choose the proposals ${ }^{11}$.

One may argue that our findings depend crucially on the symmetry assumptions. It is important, then, to clarify the role symmetry plays in our model. Let's concentrate in Assumption 1 which imposes symmetry on the distribution of the median voter ${ }^{12}$. It is true that without symmetry of $f$ no general result can be obtained. More precisely, one can easily find examples with asymmetric density function $f$ for which statement i) in the theorem doesn't hold, i.e. one party is better off in the Party Equilibrium than in the Candidate Equilibrium. This, however, doesn't mean that our results are not robust to changes in our assumption on $f$ (as long as the changes are not too big). Notice that the inequality in Theorem 3 (i) is strict, so that by continuity one could relax symmetry a little bit. A numerical example can be useful to illustrate these issues. Let's consider the utility function $u(x ; \omega) \equiv-(w-x)^{2}$ and

10 These assumptions are needed to overcome the problem of existence of equilibrium if one party is run by a candidate and the other is not (see previous footnote.)

11 In the case of competition with Downsian candidates we have supposed that can didates are free to choose the political proposals. A more realistic approach might con sider possible restrictions on candidates' choices. Parties may be able to impose some bounds on the set of policies candidates can adopt. One can show, however, that the equilibrium in this case coincides with the one in which parties compete between them selves.

12 We consider that symmetry on the function $f$ is the most critical assumption in our model. Notice that allowing for asymmetric density functions on the distribution of the median voter it is very similar to getting rid of the symmetry assumption on the loca tion of parties' ideal policy keeping the distribution of the median voter symmetric. On the other hand, symmetry on the utility of voters (and parties) it is a very common assumption on the literature and it should be seen as less problematic than symmetry of $f$. 

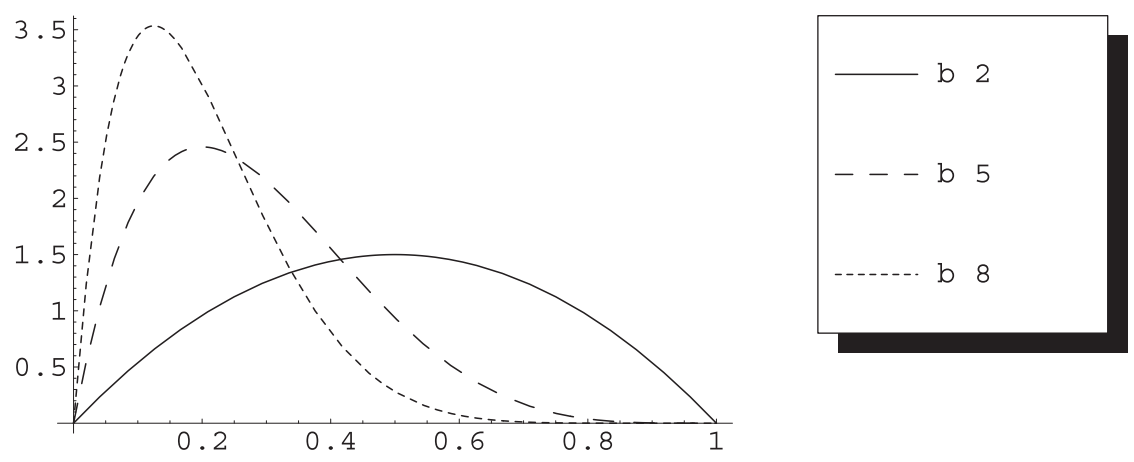

Fig. 1.

suppose that the value of the median voter is distributed according to the $\operatorname{Beta}(\mathrm{a}, \mathrm{b})$ distribution. We set the parameter $a=2$. Notice that such distribution with $b=2$ yields a symmetric density function around $\frac{1}{2}$, and the greater the value of the parameter $b$ the more concentrated the mass is near 0 . We compute the Political Equilibrium and the Candidate Equilibrium for different values of $b$ ranging from $b=2$ up to $b=8$. Figure 1 shows the Beta density function for $b=2,5,8$. For party $L$ the expected utility under the Candidate Equilibrium is always higher than the expected utility under the Party Equilibrium. For party $R$ the expected utility under the Candidate Equilibrium is higher than the expected utility under the Party Equilibrium only for values of $b$ lower than (approximately) 5 . Thus, when the parameter $b$ is greater than 5 the result provided in the theorem does not hold. Notice, however, that such distributions are highly asymmetric suggesting that our result remains valid in most realistic cases. In other words, our result is not longer valid for cases in which the median voter is, with a very high probability, very close to the ideal policy of a party and very far away from the ideal policy of the other party.

One might also wonder about the consequences of relaxing, simoultaneously, our three symmetry assumptions. Thus, consider the case where the ideal policy for party $L$ is greater than zero, the utility function is asymmetric and the density function is skewed. By the same argument given above that the inequality in Theorem 3 (i) is strict small relaxations of the symmetry assumptions are compatible with our results. A second numerical example can be useful here. Let the utility function be given by $u(x ; \omega) \equiv-\alpha(w-x)^{2}$ if $x<\omega$ and $u(x ; \omega) \equiv-(1-\alpha)(w-x)^{2}$ if $x \geq \omega$. The parameter $\alpha$ measures the degree of assymetry of the utility function (when $\alpha=0.5$ we have the symmetric case analyzed in the paper). Let the ideal policy for party $L$ be $\omega_{L}=0.1$, and let the value of the median voter be distributed according to the Beta distribution with parameters $a=2$ and $b=2.5$. Let's make $\alpha=0.55$. Thus, the utility functions, the ideal policies and the density function are not longer symmetric. However, it is not difficult to show that the inequality in Theorem 3 (i) 
still holds. Moreover, one can also show, by means of simulations ${ }^{13}$, that such inequality also holds for all values of $\omega_{L}$ in the interval $[0,0.1]$, values of $b$ in $[2,2.5]$ and values of $\alpha$ in $[0.5,0.55]$. Thus, these examples illustrate that the results are robust to small changes of the symmetry assumption.

\section{Final comments}

The results provided in our model highlight the possibility that professional politicians (the candidates) play an "insurance" role in the competition among political parties. This is more likely to happen when members of the party have the same information as candidates about the value of the median voter type. This possible insurance effect doesn't preclude, obviously, that ex-post, i.e. after the election, the party can be dissatisfied with the policy chosen by the candidate.

Two important remarks have to be made: i) We have compared the situation in which both parties choose policy versus the situation in which both candidates choose policy. One may think that who chooses policy is also part of the strategy of parties, that is, to delegate or not to delegate the power to the candidates may be seen as part of the political competition between parties. In this case, the party might design "incentive schemes" for the candidates. An analysis of these possibilities is left for future research. ii) One of the strong assumptions in the model is that the ideal policy of the expected median voter is located at the same distance from the ideal policy of party $R$ and the ideal policy of party $L$. Thus, parties are located symmetrically about the expected median voter. We believe that this is a good first approximation. In a more general model, however, one party, say $L$, may be closer to the expected median than party $R$. In this case, the first part of the theorem doesn't need to hold; it might be the case that, for enough asymmetry in the location of parties, $R$ is better off under the Party Equilibrium than under the Candidate Equilibrium when parties and candidates have the same information. The reason would be that even though the Candidate Equilibrium brings convergence in the proposals, the expected value of the lottery induced by the Party Equilibrium is much closer to $R$ than the policy outcome under the Candidate Equilibrium. A general analysis of this case is difficult since the Party Equilibrium would depend strongly on the specific type of utility function adopted. The second part of the theorem, however, would still hold: the better the information candidates have about the distribution of voters the lower the expected utility for both parties.

\section{Appendix}

Proof of Lemma 1. i) A necessary condition for $\left(l^{p}, r^{p}\right)$ to be a Political Equilibrium is that $r^{p}$ is a solution to

13 All the simulations were done with Mathematica and are available from the author upon request. 


$$
\max _{r} v_{R}\left(l^{p}, r\right)
$$

Since the median type is always an element of $[0,1]$ we have that for any $l \in \mathfrak{R}$ there exists $r^{\prime} \in[0,1]$ such that $v_{R}\left(l, r^{\prime}\right)>v_{R}(l, r)$ for all $r \notin[0,1]$. A similar argument applies for party $L$. Thus, without loss of generality, we can restrict ourselves to proposals (strategies) in the interval $[0,1]$. Recall that for $r \neq l$ we have that $u_{R}$ and $\pi(l, r)$ are differentiable in $r$. Thus the function $v_{R}(l, r)$ is differentiable at all $r \in(l, 1]$. Since $v_{R}(l, l)=u_{R}(l)$ we have

$$
v_{R}(l, r)>v_{R}(l, l) \text { for all } r \in(l, 1]
$$

Inequality (8) already shows that there cannot be a symmetric equilibrium with $l^{p}=r^{p}$.

The fact that $u_{R}^{\prime}(1)=0$ and inequality (8) imply that the solution to (7) is an element $r \in\left(l^{p}, 1\right)$ which must satisfy the first order condition

$$
0.5 f\left(\frac{l^{p}+r}{2}\right)\left(u_{R}\left(l^{p}\right)-u_{R}(r)\right)+\left(1-F\left(\frac{l^{p}+r}{2}\right)\right) u_{R}^{\prime}(r)=0
$$

which is equivalent to

$$
\frac{1-F\left(\frac{l^{p}+r}{2}\right)}{f\left(\frac{l^{p}+r}{2}\right)}=\frac{u_{R}(r)-u_{R}\left(l^{p}\right)}{2 u_{R}^{\prime}(r)}
$$

Next we show that there exists a pair $\left(l^{p}, r^{p}\right), r^{p}=1-l^{p}$, satisfying Eq. (10). Consider the expression

$$
\frac{1-F\left(\frac{r+1 r}{2}\right)}{f\left(\frac{r+1}{2}\right)}=\frac{u_{R}(r)-u_{R}(1-r)}{2 u_{R}^{\prime}(r)}
$$

The left hand side of (11) is always equal to $\frac{1 F(0.5)}{f(0.5)}=k$ which is a positive number. Let

$$
\Phi(r) \equiv \frac{u_{R}(r)-u_{R}(1-r)}{2 u_{R}^{\prime}(r)}
$$

$\Phi$ is a continuous function in the interval $[0.5,1]$ with $\Phi(0.5)=0$ and $\Phi(1)=\infty$. Thus there exists $r^{p} \geq 0.5$ such that $\Phi\left(r^{p}\right)=k$. Hence we have shown the existence of $r^{p}=1-l^{p}$ satisfying the first order condition (10). To show that $r^{p}$ is a solution to (7) it is enough to prove that there is no $r^{\prime} \neq r^{p}$ which also satisfies Eq. (10). Suppose not. Let $r^{\prime}<r^{p}$ (a similar argument works for the opposite case). Then we have

$$
\begin{aligned}
& \frac{1-F\left(\frac{l^{p}+r^{\prime}}{2}\right)}{f\left(\frac{l^{p}+r^{\prime}}{2}\right)}=\frac{u_{R}\left(r^{\prime}\right)-u_{R}\left(l^{p}\right)}{2 u_{R}^{\prime}\left(r^{\prime}\right)} \\
& \frac{1-F\left(\frac{l^{p}+r^{p}}{2}\right)}{f\left(\frac{l^{p}+r^{p}}{2}\right)}=\frac{u_{R}\left(r^{p}\right)-u_{R}\left(l^{p}\right)}{2 u_{R}^{\prime}\left(r^{p}\right)}
\end{aligned}
$$


By Assumption 2 we have

$$
\frac{1-F\left(\frac{l^{p}+r^{\prime}}{2}\right)}{f\left(\frac{l^{p}+r^{\prime}}{2}\right)} \geq \frac{1-F\left(\frac{l^{p}+r^{p}}{2}\right)}{f\left(\frac{l^{p}+r^{p}}{2}\right)}
$$

Equations (12), (13) and (14) imply that

$$
\frac{u_{R}\left(r^{\prime}\right)-u_{R}\left(l^{p}\right)}{u_{R}^{\prime}\left(r^{\prime}\right)} \geq \frac{u_{R}\left(r^{p}\right)-u_{R}\left(l^{p}\right)}{u_{R}^{\prime}\left(r^{p}\right)}
$$

But inequality (15) contradicts the fact that $u_{R}$ is concave and strictly increasing in the interval $[0,1]$.

Given that $u_{R}$ and $u_{L}$ are symmetric with respect to the point 0.5 such pair of policies $\left(l^{p}, r^{p}\right)$ is such that $l^{p}$ is the best strategy for party $L$ given that party $R$ announces $r^{p}$. Hence we have shown the existence of a symmetric party equilibrium $\left(l^{p}, r^{p}\right)$.

ii) It only remains to prove uniqueness of the symmetric equilibrium. Suppose that there exist $r$ and $r^{\prime}, r^{\prime}<r$, such that $(1-r, r)$ and $\left(1-r^{\prime}, r^{\prime}\right)$ are both symmetric equilibria. Since any equilibrium policy has to satisfy equation (11) we have

$$
\begin{aligned}
& \frac{1-F\left(\frac{1}{2}\right)}{f\left(\frac{1}{2}\right)}=\frac{u_{R}(r)-u_{R}(1-r)}{2 u_{R}^{\prime}(r)} \\
& \frac{1-F\left(\frac{1}{2}\right)}{f\left(\frac{1}{2}\right)}=\frac{u_{R}\left(r^{\prime}\right)-u_{R}\left(1-r^{\prime}\right)}{2 u_{R}^{\prime}\left(r^{\prime}\right)}
\end{aligned}
$$

Equations (16) and (17) imply

$$
\frac{u_{R}(r)-u_{R}(1-r)}{2 u_{R}^{\prime}(r)}=\frac{u_{R}\left(r^{\prime}\right)-u_{R}\left(1-r^{\prime}\right)}{2 u_{R}^{\prime}\left(r^{\prime}\right)}
$$

and this last equality contradicts the fact that $u_{R}$ is concave and strictly increasing in the interval $[0,1]$. QED.

Proof of Lemma 2. (sketch) First we show that at equilibrium candidates must propose the same policy. Suppose this is not the case and let $a^{*}<b^{*}$ (whenever it doesn't create confusion we omit the subscript). Then it is clear that policy $a^{\prime}$ such that $a^{*}<a^{\prime}<b^{*}$ increases the value of the dividing type and so we have

$$
F\left(\frac{a^{*}+b^{*}}{2} \mid I_{j}^{n}\right)<F\left(\frac{a^{\prime}+b^{*}}{2} \mid I_{j}^{n}\right)
$$

Which is a contradiction. Second we show that $a^{*}=b^{*} \neq m_{n, j}$ can not be an equilibrium. Suppose the contrary and let $a^{*}>m_{j}^{n}$. We have that $\Pi_{L}\left(a^{*}, b^{*} ; I_{j}^{n}\right)=\frac{1}{2}$. But it is clear that for all $a^{\prime}, m_{j}^{n}<a^{\prime}<a^{*}$ we have $\Pi_{L}\left(a^{\prime}, b^{*} ; I_{j}^{n}\right)>\frac{1}{2}$. Hence $\left(a^{*}, b^{*}\right)$ can not be a candidate equilibrium. It only rests to show that $a^{*}=b^{*}=m_{j}^{n}$ is a candidate equilibrium. This follows easily 
since any individual deviation would reduce the probability of winning to less than 0.5. Q.E.D.

Proof of Theorem 3. i) $\bar{v}_{L}$ is the utility for party $L$ of the lottery that with probability $1 / 2$ gives policy $l^{p}$ and with probability $1 / 2$ gives policy $r^{p}$. Recall that $l^{p}=1-r^{p}$ so that the expected value of this lottery is $1 / 2$. Now $v_{L}^{c}(1)$ is the utility that $L$ gets under the Candidate Equilibrium when the partition is $P_{1}=P_{p}=\Omega$. In this equilibrium both candidates announce the same policy $a^{*}=b^{*}=\frac{1}{2}$. Since the utility function $u_{L}$ is strictly concave it easily follows that $\bar{v}_{L}<v_{L}^{c}(1)$. The same argument works for party $R$.

ii) We need to show that

$$
v_{R}^{c}(n) \geq v_{R}^{c}(n+1)
$$

Recall that $v_{R}^{c}(n)$ is the expected utility for party $R$ when candidates have the information partition $P_{n}$, i.e., $v_{R}^{c}(n)$ is the expected utility of the "lottery" that gives $m_{i}^{n}$ with probability $e_{i}^{n} \equiv \int_{I_{i}^{n}} f(x) d x=F\left(a_{i}^{n}\right)-F\left(a_{i}^{n}{ }_{1}\right)$. Thus,

$$
v_{R}^{c}(n)=\sum_{i=1}^{2^{n} 1} e_{i}^{n} u_{R}\left(m_{i}^{n}\right)
$$

Assumption 3 implies that partition $P_{n+1}$ is such that $I_{2 i}^{n+1} \cup I_{2 i}^{n+1}=I_{i}^{n}$. Take an interval $I_{i}^{n} \subseteq\left[0, \frac{1}{2}\right]$. Note that in this case $a_{i 1}^{n}=a_{2 i}^{n+1}$ and $a_{i}^{n}=a_{2 i}^{n+1}$. By Assumption $1 f$ is increasing on the interval $\left[0, \frac{1}{2}\right]$. Hence $F$ is convex on $\left[0, \frac{1}{2}\right]$ and $F^{1}$ concave on $\left[0, \frac{1}{2}\right]$. Then we have that for all $\mu \in[0,1]$

$$
\begin{aligned}
\mu F^{1} & \left(\frac{F\left(a_{2 i}^{n+1}\right)+F\left(a_{2 i}^{n+1}\right)}{2}\right)+(1-\mu) F^{1}\left(\frac{F\left(a_{2 i}^{n+1}\right)+F\left(a_{2 i}^{n+1}\right)}{2}\right) \\
& \leq F^{1}\left(\mu \frac{F\left(a_{2 i}^{n+1}\right)+F\left(a_{2 i}^{n+1}\right)}{2}+(1-\mu) \frac{F\left(a_{2 i}^{n+1}\right)+F\left(a_{2 i 1}^{n+1}\right)}{2}\right)
\end{aligned}
$$

Take $\mu:=\frac{F\left(a_{2 i}^{n+1}\right) F\left(a_{22}^{n+1}\right)}{F\left(a_{2 i}^{n+1}\right) F\left(a_{2 i}^{n+1}\right)}$. It is always true that $\mu \in[0,1]$, then the right hand side of (18) is equal to

$$
\begin{gathered}
F^{1}\left(\frac{F\left(a_{2 i}^{n+1}\right)+F\left(a_{2 i}^{n+1}\right)}{2}+\frac{\mu}{2}\left(F\left(a_{2 i}^{n+1}\right)-F\left(a_{2 i}^{n+1}\right)\right)\right) \\
=F^{1}\left(\frac{F\left(a_{2 i}^{n+1}\right)+F\left(a_{2 i}^{n+1}\right)}{2}\right) \\
=F^{1}\left(\frac{F\left(a_{i}^{n}\right)+F\left(a_{i}^{n} 1\right)}{2}\right)=m_{i}^{n}
\end{gathered}
$$

Note that

$$
\begin{aligned}
& F^{1}\left(\frac{F\left(a_{2 i}^{n+1}\right)+F\left(a_{2 i}^{n+1} 2\right)}{2}\right)=m_{2 i}^{n+1} 1 \\
& F^{1}\left(\frac{F\left(a_{2 i}^{n+1}\right)+F\left(a_{2 i}^{n+1} 1\right)}{2}\right)=m_{2 i}^{n+1}
\end{aligned}
$$


and

$$
\mu=\frac{e_{2 i}^{n+1} 1}{e_{i}^{n}}
$$

Then inequality (18) can be written as

$$
\frac{e_{2 i}^{n+1} 1}{e_{i}^{n}} m_{2 i}^{n+1} 1+\left(1-\frac{e_{2 i}^{n+1} 1}{e_{i}^{n}}\right) m_{2 i}^{n+1} \leq m_{i}^{n}
$$

By Assumption $1 f$ is decreasing on the interval $\left[\frac{1}{2}, 1\right]$. Hence, the same argument as above can be used to show that for any interval $I_{i}^{n} \subseteq\left(\frac{1}{2}, 1\right]$ we have

$$
\frac{e_{2 i}^{n+1} 1}{e_{i}^{n}} m_{2 i}^{n+1}+\left(1-\frac{e_{2 i}^{n+1} 1}{e_{i}^{n}}\right) m_{2 i}^{n+1} \geq m_{i}^{n}
$$

It is not difficult to see that symmetry of $f$ around $\frac{1}{2}$ and part ii) in Assumption 1 imply that

$$
e_{i}^{n}=e_{o(i)}^{n}
$$

and

$$
m_{i}^{n}=1-m_{o(i)}^{n}
$$

Thus we can write

$$
v_{R}^{c}(n)=\sum_{i=1}^{(1 / 2) 2^{n} 1} e_{i}^{n}\left[u_{R}\left(m_{i}^{n}\right)+u_{R}\left(m_{o(i)}^{n}\right)\right]
$$

and

$$
\begin{aligned}
v_{R}^{c}(n+1)= & \sum_{i=1}^{(1 / 2) 2^{n} 1} e_{2 i}^{n+1}\left[u_{R}\left(m_{2 i}^{n+1}\right)+u_{R}\left(m_{o(2 i}^{n+1} 1\right)\right] \\
& +e_{2 i}^{n+1}\left[u_{R}\left(m_{2 i}^{n+1}\right)+u_{R}\left(m_{o(2 i)}^{n+1}\right)\right]
\end{aligned}
$$

To prove that $v_{R}^{c}(n) \geq v_{R}^{c}(n+1)$ is enough to show that each term in Eq. (22) is greater (or equal) than the corresponding term in Eq. (23), i.e., it is enough to show that for all $i=1, \ldots, \frac{1}{2} 2^{n} \quad 1$

$$
\begin{aligned}
e_{i}^{n}\left[u_{R}\left(m_{i}^{n}\right)+u_{R}\left(m_{o(i)}^{n}\right)\right] \geq & e_{2 i}^{n+1}\left[u_{R}\left(m_{2 i}^{n+1}\right)+u_{R}\left(m_{o(2 i}^{n+1} 1\right)\right] \\
& +e_{2 i}^{n+1}\left[u_{R}\left(m_{2 i}^{n+1}\right)+u_{R}\left(m_{o(2 i)}^{n+1}\right)\right]
\end{aligned}
$$

Since

$$
e_{2 i}^{n+1}=e_{i}^{n}-e_{2 i 1}^{n+1}
$$

inequality (24) is equivalent to

$$
e_{i}^{n}\left[u_{R}\left(m_{i}^{n}\right)+u_{R}\left(m_{o(i)}^{n}\right)\right] \geq A
$$


where

$$
\begin{aligned}
A \equiv & e_{2 i}^{n+1}\left[u_{R}\left(m_{2 i}^{n+1}\right)+u_{R}\left(m_{o(2 i}^{n+1}\right)\right] \\
& +\left(e_{i}^{n}-e_{2 i}^{n+1}\right)\left[u_{R}\left(m_{2 i}^{n+1}\right)+u_{R}\left(m_{o(2 i)}^{n+1}\right)\right]
\end{aligned}
$$

Let

$$
\begin{aligned}
B \equiv & e_{i}^{n} u_{R}\left(\frac{e_{2 i}^{n+1} 1}{e_{i}^{n}} m_{2 i}^{n+1} 1+\frac{e_{i}^{n}-e_{2 i}^{n+1} 1}{e_{i}^{n}} m_{2 i}^{n+1}\right) \\
& +e_{i}^{n} u_{R}\left(\frac{e_{2 i}^{n+1} 1}{e_{i}^{n}} m_{o(2 i}^{n+1} 1\right) \\
& \left.+\frac{e_{i}^{n}-e_{2 i}^{n+1}}{e_{i}^{n}} m_{o(2 i)}^{n+1}\right)
\end{aligned}
$$

Concavity of $u_{R}$ implies

$$
B \geq \frac{e_{i}^{n}}{e_{i}^{n}} A=A
$$

The fact that for all $j$ and $n$ we have $m_{j}^{n}=1-m_{o(j)}^{n}$ implies that

$$
\begin{aligned}
m_{i}^{n}+m_{o(i)}^{n}= & \frac{e_{2 i}^{n+1} 1}{e_{i}^{n}}\left(m_{2 i}^{n+1}+m_{o(2 i}^{n+1}\right) \\
& +\frac{e_{i}^{n}-e_{2 i}^{n+1}}{e_{i}^{n}}\left(m_{2 i}^{n+1}+m_{o(2 i)}^{n+1}\right)
\end{aligned}
$$

Hence, concavity of $u_{R}$, equality (26) and inequalities (20), (21) and (25) imply

$$
e_{i}^{n}\left[u_{R}\left(m_{i}^{n}\right)+u_{R}\left(m_{o(i)}^{n}\right)\right] \geq B \geq A
$$

and we conclude that (24) holds. Q.E.D.

\section{References}

[1] Alesina A (1988) Credibility and policy convergence in a two party system with rational voters. Am Econ Rev 78: 796806

[2] Alesina A, Rosenthal H (1996) A theory of divided government. Econometrica 64(6): 13111341

[3] Alesina A, Rosenthal H (1995) Partisan politics, divided government, and the economy. Cambridge University Press, New York

[4] Barlow RE, Proschan F (1981) Statistical theory of reliability and life testing. To Begin With, Silver Spring, MD

[5] Calvert R (1985) Robustness of the multidimensional voting model: candidate motivations, uncertainty and convergence. Am J Pol Sci 29: 6995

[6] Caplin A, Nalebuff B (1991) Aggregation and social choice: a mean voter theo rem. Econometrica 59(1): 123

[7] Cox GW (1987) Electoral equilibrium under alternative voting institutions. Am J Pol Sci 31: 82108

[8] Denzau A, Kats A, Slutsky S (1985) Multi Agent equilibria with market share and ranking objectives. Soc Choice Welfare 2: 95117

[9] Downs A (1957) An economic theory of democracy. Harper Collins, New York 
[10] Eaton BC, Lipsey R (1975) The principle of minimum differentiation reconsidered: some new developments in the theory of spatial competition. Rev Econ Stud 42: 2750

[11] Hansson I, Stuart C (1984) Voting competitions with interested politicians: Plat forms do not converge to the preferences of the median voter. Publ Choice 44: 431441

[12] Ortuno Ortín I (1997) A spatial model of political competition and proportional representation. Soc Choice Welfare 14: 427438

[13] Osborne MJ (1995) Spatial model of political competition under plurality rule: a survey of some explanations of the number of candidates and the position they take. Can J Econ XXVIII (2): 261301

[14] Pratt J (1981) Concavity of the log likelihood. J Am Stat Assoc 76(373): 103106

[15] Roemer JE (1994) A theory of policy differentiation in single issue electoral poli tics. Soc Choice Welfare 11: 335380

[16] Roemer JE (1997) Political economic equilibrium when parties represent con stituents: The unidimensional case. Soc Choice Welfare 14: 479502

[17] Wittman D (1983) Candidate motivation: a synthesis of alternative theories. Am Pol Sci Rev 77: 142157

[18] Wittman D (1990) Spatial strategies when candidates have policy preferences. In: Enelow J, Hinich M (eds) Advances in the spatial theory of voting. Cambridge University Press, Cambridge 